\title{
Comparative Study of Lipoprotein (A) Level in Type 2 Diabetics and Non Diabetics with Acute Coronary Syndrome
}

\author{
Akila Selvam, ${ }^{1}$ Prabhakar ${ }^{2}$ \\ ${ }^{1}$ Assistant Professor, Department of General Medicine, Thiruvannamalai Medical College, Thiruvannamalai. \\ ${ }^{2}$ Associate Professor, Department of General Medicine, Thiruvannamalai Medical College, Thiruvannamalai.
}

\begin{abstract}
Objective: To compare the level of lipoprotein (a) level in type 2 diabetics and non diabetics in association with acute coronary syndrome.

Materials And Methods: This cross-sectional study was conducted on patients referred to ICU with acute coronary syndrome in General Medicine department, Thiruvannamalai Medical College. Hundred subjects including 50 diabetics as test group and 50 non diabetics as control group who were matched for age and sex were enrolled in this study. We measured serum FBS, TG, cholesterol, LDL, HDL and serum Lp (a) in both groups. Data were analyzed by T-test and chi-square.

Results: Lp (a) level was significantly higher in diabetics when compared with control group (Diabetic group $27.62 \pm 12.80 \mathrm{vs}$ non diabetic group $12.5 \pm 5.42 \mathrm{mg} / \mathrm{dl}$ ).
\end{abstract}

\section{Introduction}

Cardiovascular disease has emerged as major health problem worldwide. Coronary artery disease $(\mathrm{CAD})$ has a multi factorial origin, including hereditary and acquired risk factors which may be the direct cause of the disease or merely associated with it. Changes in lipid metabolism play a relevant role in the progression of atherosclerosis and the laboratory assessment of lipoproteins is of fundamental importance to diagnose and treat this condition.

In Indians more than 60 percent CAD remains unexplained by conventional risk factors. In this setting newer cardiovascular risk factors are of great importance. Newer risk factors which are elevated in Indians in comparative studies include lipoprotein(a), CRP and homocysteine levels. Lipoprotein(a) level is consistently elevated in Indians compared to other ethnic groups. ${ }^{(1)}$ High levels of lipoprotein (a) [Lp(a)] are known to be a cardiovascular risk factor associated with premature coronary artery disease

Type 2 diabetes mellitus (DM) is associated with significant cardiac morbidity and mortality with a more than threefold increased risk of coronary artery disease (CAD). However, this increased cardiovascular risk is only partially explained by conventional risk factors like hypertension, lipid abnormalities, central obesity and glucose intolerance. Lipoprotein (a) (Lp (a)) has been established as an independent risk factor for CAD. ${ }^{(2)}$. Therefore, this study was designed to compare the serum Lp (a) level in patients with type 2 diabetes and nondiabetic individuals.

\section{Methodology}

The study was conducted during the period of September 2015 to March 2016 among patients admitted with acute coronary syndrome in cardiac intensive care unit, government Thiruvannamalai Medical College, Thiruvannamalai. Approval from institutional ethical committee was obtained before starting the study. Informed consent was obtained from all patients before enrolling them for the study.

Fifty Type 2 diabetic patients and fifty non diabetic patients admitted with acute coronary syndrome were selected after matching for age and sex, during the study period. Lipoprotein (a) level was studied in these patients.

\section{Inclusion Criteria}

Patients with angina, occurring at rest (CCS class IV) of duration more than 30 minutes, but within 24 hours from onset of angina were included in the study. Among them, fifty patients had type 2 diabetes. Fifty age and sex matched non diabetic were included as the other group. Diabetes was ruled out in non diabetic group with fasting and 2-hr post prandial blood glucose measurement.

\section{Exclusion Criteria}

The following patients were excluded from the study:

$>$ Patients presenting $24 \mathrm{hrs}$ after occurrence of angina

> Angina secondary to extra cardiac causes. (Eg. Anaemia, thyrotoxicosis) 
> Patients who were currently on drugs which may alter serum levels of Lp (a) (Eg. Oral contraceptive pills, cholesterol lowering agents 1 , aspirin, hormone replacement therapy, antiepileptics). ${ }^{(3,4,5)}$

Prediabetic patients were excluded from both groups. ${ }^{(6)}$

> All type of renal failure patients including diabetic nephropathy. ${ }^{(7)}$

\section{Method Of Collection Of Data}

In the present study hundred acute coronary syndrome patients, who were divided in to two separate age and sex matched groups; one with type 2 diabetes mellitus and one without Diabetes mellitus, were selected by stratified sampling, during the study period as per the inclusion and exclusion criteria.

A detailed history (with emphasis on angina/angina equivalent characteristics) was taken and a detailed physical examination was done as per the proforma to study the clinical profile of patients, blood parameters and imaging as required.

All diabetics are of type 2 diabetes mellitus, currently on oral hypoglycemic agents. In non diabetic group diabetes mellitus was ruled out by fasting and post prandial blood glucose which was analysed by GODPAP(glucose oxidase-phenol 4-aminophenazone peroxidase)method. In all 100 patients serum Lp(a) level was estimated.

Resting electrocardiogram with 12 standard leads was taken as soon as possible and repeated serially and ST segment was monitored. Based on the findings both diabetic and non diabetic groups of patients were regrouped.

Other relevant investigations are done

- ECG in all leads,Haemoglobin\%,Total count, Erythrocyte sedimentation rate

- Blood sugar at the time of admission, fasting and $2 \mathrm{hr}$ blood sugar

- Blood urea, Serum creatinine, Serum Lipoprotein (a),Total cholesterol,HDL level,LDL level,Triglycerides,Echocardiogram

\section{Statistical Analysis}

Statistical analysis was done by using percentages, mean values, standard deviation, chi-square test, t-test and proportion test. A p-value $<0.05$ level was considered statistically significant and a p-value $>0.05$ was considered as not statistically significant.

\section{Observations And Results}

Table I: Age And Sex Wise Distribution Of Cases:

\begin{tabular}{|l|l|l|l|l|l|l|l|}
\hline & \multicolumn{3}{|l|}{ DIABETIC } & \multicolumn{4}{l|}{ NON DIABETIC } \\
\hline S. NO. & AGE & MALE & FEMALE & TOTAL & MALE & FEMALE & TOTAL \\
\hline 1 & $21-30$ & 4 & 0 & 4 & 4 & 0 & 4 \\
\hline 2 & $31-40$ & 7 & 3 & 10 & 4 & 1 & 5 \\
\hline 3 & $41-50$ & 4 & 5 & 9 & 5 & 7 & 12 \\
\hline 4 & $51-60$ & 4 & 12 & 16 & 7 & 6 & 13 \\
\hline 5 & $61-70$ & 3 & 5 & 8 & 1 & 9 & 10 \\
\hline 6 & $71-80$ & 2 & 0 & 2 & 2 & 2 & 4 \\
\hline 7 & $81-90$ & 1 & 0 & 1 & 2 & 0 & 2 \\
\hline & & 25 & 25 & 50 & 25 & 25 & 50 \\
\hline
\end{tabular}

The following observations are made from above chart a. Majority of patients belongs to the age group of 51-60 years of age.

b. There is no significant difference in age wise distribution of cases in both groups, so they are comparable $\mathrm{p}<0.05$

Table II: Age Wise Distribution Of Lipoprotein (A) Level In Diabetic Group:

\begin{tabular}{|l|l|l|l|l|l|l|l|}
\hline S.NO. & Lp(a) levels & \multicolumn{6}{|l|}{ AGE IN YEARS } \\
\hline & & $21-30$ & $31-40$ & $41-50$ & $51-60$ & $61-70$ & $>71$ \\
\hline 1 & $0-15$ & 1 & 2 & 1 & 4 & 2 & 1 \\
\hline 2 & $16-30$ & 1 & 6 & 2 & 7 & 4 & 1 \\
\hline 3 & $31-45$ & 2 & 2 & 3 & 4 & 1 & 1 \\
\hline 4 & $46-60$ & 0 & 0 & 3 & 2 & 0 & 0 \\
\hline & TOTAL & 4 & 10 & 9 & 17 & 7 & 3 \\
\hline
\end{tabular}




\section{AGE WISE DISTRIBUTION OF Lp(a) in diabetics groups}

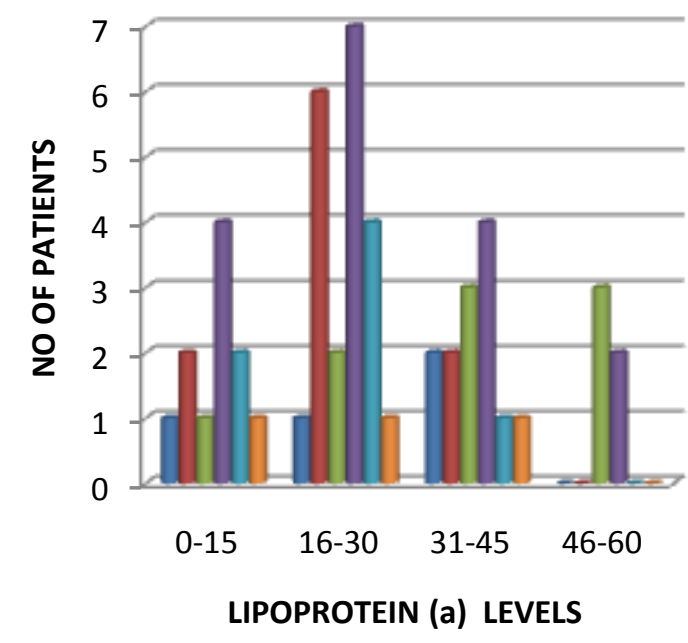

- AGE WISE

DISTRIBUTION OF Lp(a)

IN DIABETIC GROUPS

LP(a) levels 21-30

AGE WISE

DISTRIBUTION OF Lp(a)

IN DIABETIC GROUPS

AGE IN YEARS 31-40

following observations was made from above chart:

A. The maximum value of $\mathrm{Lp}$ (a) level seen in 51-60 age group.

B. The minimum value of $\mathrm{Lp}$ (a) level seen in 21-30 age group.

C. Ninty percent of diabetic patients have Lp (a) levels more than $15 \mathrm{mg} / \mathrm{dl}$.

D.Thirty two percent of diabetic patients have Lp(a) level more than $30 \mathrm{mg} / \mathrm{dl}$.

Table III: Age Wise Distribution Of Lp(A) In Non-Diabetic Groups

\begin{tabular}{|l|l|l|l|l|l|l|l|}
\hline S.NO. & Lp(a) levels & AGE IN YEARS & \multicolumn{1}{l|}{ IN1 } \\
\hline & & $21-30$ & $31-40$ & $41-50$ & $51-60$ & $61-70$ & $\mathbf{6}$ \\
\hline $\mathbf{1}$ & $\mathbf{0 - 1 5}$ & $\mathbf{2}$ & $\mathbf{5}$ & $\mathbf{9}$ & $\mathbf{1 2}$ & $\mathbf{9}$ & $\mathbf{6}$ \\
\hline $\mathbf{2}$ & $\mathbf{1 6 - 3 0}$ & $\mathbf{2}$ & $\mathbf{0}$ & $\mathbf{3}$ & $\mathbf{1}$ & $\mathbf{1}$ & $\mathbf{0}$ \\
\hline $\mathbf{3}$ & $\mathbf{3 1 - 4 5}$ & $\mathbf{0}$ & $\mathbf{0}$ & $\mathbf{0}$ & $\mathbf{0}$ & $\mathbf{0}$ & $\mathbf{0}$ \\
\hline $\mathbf{4}$ & $\mathbf{4 6 - 6 0}$ & $\mathbf{0}$ & $\mathbf{0}$ & $\mathbf{0}$ & $\mathbf{0}$ & $\mathbf{0}$ & $\mathbf{0}$ \\
\hline & TOTAL & $\mathbf{4}$ & $\mathbf{5}$ & $\mathbf{1 2}$ & $\mathbf{1 3}$ & $\mathbf{1 0}$ & $\mathbf{6}$ \\
\hline
\end{tabular}

The following observation was made from above chart:

a. Most of the non diabetic group patients have Lp(a) level of less than $15 \mathrm{mg} / \mathrm{dl}$.

b. Only $10 \%$ of non diabetic group have value more than $15 \mathrm{mg} / \mathrm{dl}$.

Table IV: Comparison Of Lp(A) Level In Diabetic And Non Diabetic Group:

\begin{tabular}{|l|l|}
\hline GROUP & MEAN(S.D) $(\mathbf{m g} / \mathbf{d l})$ \\
\hline Type 2 Diabetic & $27.62 \pm 12.5$ \\
\hline Non diabetic & $12.5 \pm 5.42$ \\
\hline
\end{tabular}

Comparison Of Lp(A) Level In Diabetic And Non Diabetic Group:

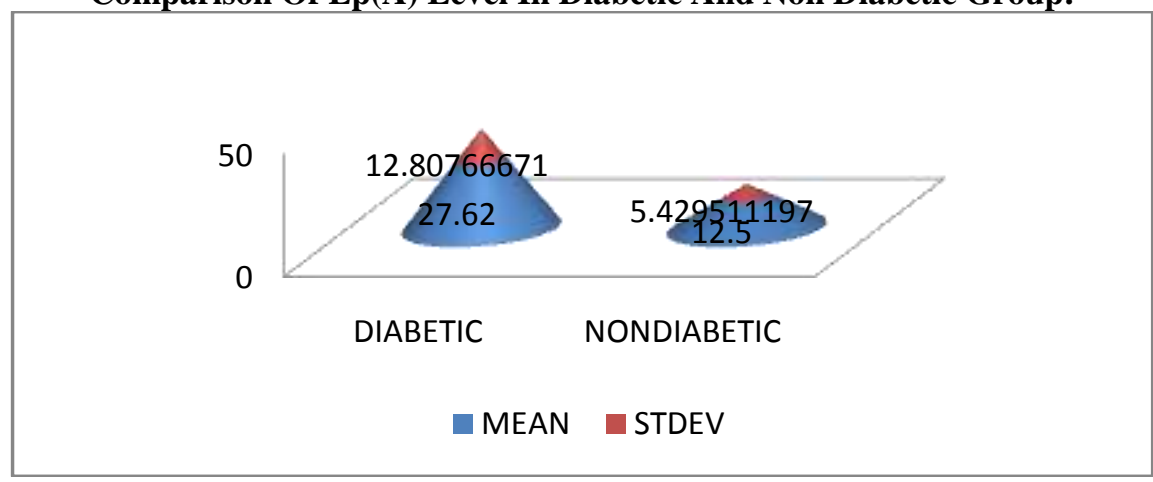


Comparative Study Of Lipoprotein (A) Level In Type 2 Diabetics And Non Diabetics With Acute ..

Difference between means of serum Lp(a) level in both groups is significant $(\mathrm{p}<0.001)$. Type 2 diabetic patients had a higher Lp(a) levels compared to non diabetics with acute coronary syndrome.

Table V: Comparing Lp(A) Level And Lipid Profile In Both Group With Acute Coronary Syndrome:

\begin{tabular}{|l|l|l|l|l|}
\hline S.NO & PROFILE & DIABETICS & NON DIABETICS & p VALUE \\
\hline & & & & \\
\hline 1 & LDL & $114 \pm 19.79$ & $101.2 \pm 11.65$ & $<0.001$ \\
\hline 2 & TOTALCHOLESTEROL & $188.4 \pm 26.15$ & $161 \pm 21.13$ & $<0.001$ \\
\hline 3 & TRIGLYCERIDES & $193.72 \pm 34.39$ & $140 \pm 16.37$ & $<0.001$ \\
\hline 4 & HDL LEVEL & $29.02 \pm 10.42$ & $48.3 \pm 11.20$ & $<0.001$ \\
\hline 5 & Lp(a) LEVEL & $27.62 \pm 12.80$ & $12.5 \pm 5.42$ & $<0.001$ \\
\hline
\end{tabular}

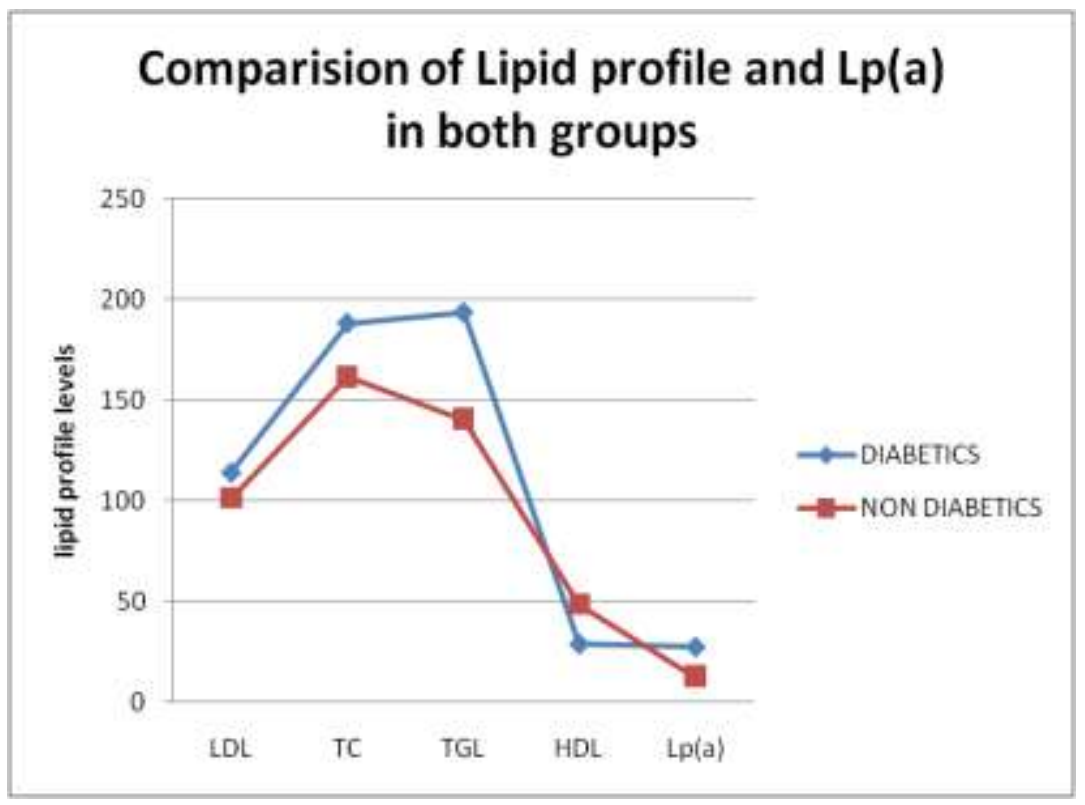

Serum Lp(a) level, Total cholesterol, Triglycerides, LDL were significantly higher in type 2 diabetic group when compared to non diabetic group $(\mathrm{p}<0.001)$.HDL level was significantly lower in type 2 diabetic group than non diabetic group $(\mathrm{p}<0.001)$.

Table VI: Odds Ratio

\begin{tabular}{|l|l|l|}
\hline Number of cases & $\mathrm{Lp}(\mathrm{a})>15 \mathrm{mg} / \mathrm{dl}$ & $\mathrm{Lp}(\mathrm{a})<15 \mathrm{mg} / \mathrm{dl}$ \\
\hline Diabetic group & 39 & 11 \\
\hline Non diabetic group & 7 & 43 \\
\hline
\end{tabular}

Odds Ratio $=21.77$

95\% Cl 7.6822 To 61.7449

Z Statistic 5.795

P Value $<0.0001$

Relative Risk $=4.16$

95\% Cl 2.4221 To 7.1519

Z Statistic 5.163

P Value $<0.0001$

Diabetic group had an odds ratio of 21.7 when compared to non diabetic group, in having Lp(a) levels $>15 \mathrm{mg} / \mathrm{dl}$. There is a positive correlation of serum LP(a) levels with LDL and triglycerides level.

\section{Discussion}

In Asian Indians, the coronary artery disease rate has been raising rapidly. ${ }^{(8)}$ The cause for its high prevalence of coronary artery disease in Asian Indians has been largely unexplained. It is due to the failure of traditional risk factors to explain which raises the possibility of a genetic susceptibility to coronary disease in Asian Indians. 
So, studies on lipoprotein(a) levels are significant as lipoprotein(a) is known to be genetically determined. As $\mathrm{Lp}$ (a) values are different in various ethnic group populations, but in most of the studies revealed that south Asians have higher level of $\mathrm{Lp}(\mathrm{a})$ concentrations. It is proved by Enas et al study. ${ }^{(1)}$ Similar to this, the present study also showed that $\mathrm{Lp}(\mathrm{a})$ levels are increased in in our population.

In diabetes mellitus, $67 \%$ of deaths are due to coronary artery disease. Framingham heart study showed that CAD, acute myocardial infarction and sudden death is $1-5$ fold increased in diabetes patients. ${ }^{(9)}$ So, diabetic patients with coronary artery disease have worst prognosis when compared to non diabetic patient with CAD. Diabetes also predisposes to early thrombus, smooth muscle proliferation, endothelial and platelet dysfunction.

In the present study, hundred patients with acute coronary syndrome were taken, of which 50 patients are diabetic and 50 patients non diabetic. Serum Lp(a) was compared between these two groups, along with comparison of other parameters.

The two groups were selected from cases of acute coronary syndrome admitted at cardiac intensive care unit Tiruvannamalai Medical College, Tiruvannamalai between September 2015 and March 2016. Both groups have equal number of cases and were age and sex matched for uniform comparison.

Comparison Of Clinical Profile In Various Studies

\begin{tabular}{|c|c|c|c|c|}
\hline STUDY & NO & AGE & MALE/FEMALE & PROPORTION \\
\hline 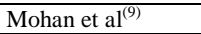 & 100 & $54 \pm 8.1$ & $72 / 28$ & 1 \\
\hline 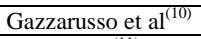 & 103 & $56.6 \pm 2.3$ & $62 / 13$ & 1.12 \\
\hline Tseng et $\mathrm{al}^{(11)}$ & 556 & $63.4 \pm 10.4$ & $43.6 / 56.4$ & 1.02 \\
\hline Salehi et al $^{(12)}$ & 115 & $58.7 \pm 13$ & $31 / 10$ & 1.2 \\
\hline Present study & 100 & $50.82 \pm 13$ & $50 / 50$ & 1 \\
\hline
\end{tabular}

The mean age of the cases studied in the present study is comparatively younger $(\mathrm{p}>0.05)$ than similar studies, except Mohan et al, with which the age is comparable. ${ }^{(9)}$ This may be due to occurrence of acute coronary syndrome in an earlier age in the South Indian patients compared to western population.

The ratio between male and female patients in both controls and cases were proportionate in all the studies. Gender bias is also removed as both the groups are matched.

Post-menopausal state influences serum Lp(a) levels. Both the diabetic and non- diabetic group had comparable fraction of such patients. Females on Hormone replacement therapy were also excluded from the study as it alters athero thrombotic risk and $\mathrm{Lp}(\mathrm{a})$ levels.

Comparison Of Lp(A) Level In Type -2 Diabetics And Non Diabetics In Various Studies

\begin{tabular}{|c|c|c|c|}
\hline Author & No. of cases & Lp(a) level in diabetics & Lp(a) level in non diabetics \\
\hline Mohan et al ${ }^{(9)}$ & 100 & $15.1 \pm 3.3$ & $12.1 \pm 4.6$ \\
\hline Tseng et $\mathrm{al}^{(11)}$ & 557 & $18.1 \pm 15.5$ & $14.9 \pm 5.9$ \\
\hline Neki et al ${ }^{(13)}$ & 134 & $14.9 \pm 3.5$ & $11.9 \pm 4.1$ \\
\hline${\text { Salehi et } \mathrm{al}^{(12)}}^{(12)}$ & 115 & $16.8 \pm 4.4$ & $13.3 \pm 5.1$ \\
\hline Present study & 100 & $27.62 \pm 12.8$ & $12.5 \pm 5.42$ \\
\hline
\end{tabular}

In the present study, $\mathrm{Lp}$ (a) values varied from 7 to $60 \mathrm{mg} / \mathrm{dl}$. Type-2 diabetic patients had higher Lp(a) level compared to non diabetic patients. The difference of means was statistically significant $(\mathrm{p}<0.0001)$. This was comparable with Mohan et al, Neki et al, and Salehi et al. ${ }^{(9,12,13)}$ This was because of similar patient sample and method used to measure serum $\mathrm{Lp}(\mathrm{a})$ level.

In the present study, considering the optimal cut off points at $15 \mathrm{mg} / \mathrm{dl}, 80 \%$ of type- 2 diabetic patients had $\mathrm{Lp}(\mathrm{a})>15 \mathrm{mg} / \mathrm{dl}$, compared to $20 \%$ of non diabetic patients.

Type-2diabetic patients, have nearly 2 fold risk of higher serum lipoprotein(a) compared to non diabetic group, during acute coronary syndromes.

This could be explained by 2 hypothesis:

A) Diabetic group might have had higher pre existing base line serum Lp(a) level and this could have contributed to the pre mature acute coronary syndrome.

B) Acute coronary syndrome might have caused an accelerated acute phase reaction in the diabetic group. Thus it may be a prognostic marker in such situations.Long term prospective studies analysing lipoprotein(a) levels with other parameters like plasma insulin, C-peptide levels and HbAlc levels are needed to confirm the hypothesis.

\section{Lp(a) level and lipid profile}

In the present study, serum total cholesterol, LDL level, triglycerides and Lp(a) level were significantly higher $(\mathrm{p}<0.0001)$ in the diabetic group when compared to non diabetic groups. HDL are significantly lower in diabetic group when compared to non diabetic groups. In the present study, there was moderate positive correlation between serum Lp(a) levels and serum total cholesterol and LDL. This was 
consistent with Mohan et al. ${ }^{(9)}$ Raised Lp(a) can increase the coronary artery disease risk along with raised LDL in type-2 diabetes. This suggests the basis for a genetic pre disposition to coronary artery disease among populations with elevated Lp(a) levels and concomitantly raised LDL levels.

\section{Morbidity And Mortality}

Diabetic patients with acute myocardial infarction have a higher mortality. Since the present study had a small sample and is a cross sectional study, this result cannot be concluded. In the present study, cardiac arrest occurred in four diabetic patients because of secondary ventricular fibrillation out of whom only one survived. Cardiac arrest occurred in one non-diabetic patient, who succumbed to a sustained pulseless ventricular tachycardia..

\section{Summary}

$>$ Serum Lp(a) level is high in type -2 diabetic patients with acute coronary syndrome when compared to non diabetic patients.

$>$ Mean serum Lp(a) in present study was $27.62 \pm 12.8 \mathrm{mg} / \mathrm{dl}$. The value of $\mathrm{Lp}$ (a) varies from $7 \mathrm{mg} / \mathrm{dl}$ to $60 \mathrm{mg} / \mathrm{dl}$.

$>$ Triglycerides, total cholesterol and LDL were significantly higher in patients with type -2 diabetics and acute coronary syndrome compared to non diabetic patients.

$>$ HDL cholesterol levels were significantly lower in patients with type -2 diabetic patients when compared to non diabetic group with acute coronary syndrome.

\section{Conclusions}

$>$ Type-2 diabetic patients have higher level of Lp(a) level during acute coronary syndrome when compared to non diabetic group. So, the type-2 diabetic patients have nearly 2 fold risk of having high Lp(a) level than non diabetic group.

$>$ Elevated Lp(a) in type -2 diabetic patients contribute to the accelerated atherogenic / prothrombotic state, causing major adverse cardiac events.

\section{References}

[1]. Enas GG, Dahlen G, Berg K et al. Lp(a) lipoprotein as a risk factor for myocardial infarction. J Am Med Assoc. 1986;256: 2540-44.

[2]. Scanu AM. Lipoptotein(a) and the antithrombotic process: mechanistic insightsand clinical implications. Curr Atherosclr Rep 2003;5:106-13

[3]. Utermann G: The mysteries of lipoprotein(a). Science1989; 246:904-910.

[4]. Kronenberg F, Steinmetz A, kostner GM and Dieplinger H: Lipoprotein in health and disease. Crit Rev Clin Lab Sci 1996; 33:495543.

[5]. Gurakar A, Hoeg JM, Kostner G et al. Levels of lipoprotein Lp(a) decline with neomycin and niacin treatment. Atherosclerosis. $1985 ;$ 57:293-301.

[6]. World Health Organisation: Diabetes Mellitus: Report of a WHO study group. Geneva, World Health Org,.1996(Tech Reo Ser No. $855)$.

[7]. Bostem AG, Cupples LA, Srivastava LM. Lipoprotein (a): Biology and role in atherosclerotic vascular disease. Current Science 1999;76:1553-60.

[8]. Haffner SM: Lipoprotein(a) and diabetes. Diabetes care 1993; 16:835-840.

[9]. Mohan V, Deepa R, Harnath SP et al. Lipoprotein(a) is an independent risk factor for coronary artery disease in NIDDM patients in south India. Diabetes Care 1998;21:1819-23.

[10]. Gazzaurssco C, Garzaniti S, Giordaneti et alassessment of asymptomatic coronary artery disease in apparently uncomplicated type 2 diabetes patients - A role lipoprotein(a) and apolipoprotein(a) polymorphism. Diabetes Care 2002;25:1418-1424.

[11]. Tseng CH, Chong CK, Chen CJ, Tai TY: Lipoprotein(a) is an independent risk factorfor peripheral arterial disease in Chinese type 2 diabetes patients in Taiwan. Diabetes Care 2004;27:517-522.

[12]. Salehi R, Rahbani MN, Rashtchizadeh N. Lipoprotein(a), apo(a) phenotypes and dyslipidemia in acute coronary syndrome. Med J Islam Repub Iran 2001;14(4):139-143.

[13]. Neki AD, Durrington PN, Serum Lp(a) lipoprotein concentration and outcome of thrombolytic treatment for myocardial infarction. Br Heart J. 1994;71:316-321. 